\title{
Sliding Mode Control of Uncertain Systems with Arbitrary Relative Degree and Unknown Control Direction: Theory and Experiments.
}

\author{
Alessandro Jacoud Peixoto, Tiago Roux Oliveira and Liu Hsu
}

\begin{abstract}
This paper addresses the design of output feedback sliding mode tracking control for single-input-singleoutput (SISO) uncertain linear plants with arbitrary relative degree. A monitoring function is used to cope with the lack of knowledge of the control direction, i.e., the high frequency gain sign. The proposed scheme guarantees global asymptotic stability with respect to a compact set. Moreover, asymptotically exact output tracking is obtained. To this end, a key element is a hybrid nonlinear filter which performs exact signal differentiation based on higher order sliding modes. The proposed controller is evaluated with a DC motor control experiment.
\end{abstract}

Keywords: uncertain systems, output feedback, control direction, exact tracking, higher order sliding mode.

\section{INTRODUCTION}

The control of uncertain plants with unknown control direction, is a classical problem in the adaptive control literature. The Nussbaum gain has been played a major role in this context [2], [3], however, it is of arguable practical interest, due to the large transients and lack of robustness that may result [3][4]. In contrast to the adaptive control literature, few publications are available in the domain of sliding mode control (SMC) for this class of plants. In [5], a SMC was proposed for a quite general class of uncertain nonlinear systems. However, state feedback was required. In [6], a hybrid scheme was proposed for uncertain first order nonlinear systems. It was argued that the proposed scheme could avoid the large transient resulting from the Nussbaum gain approach. An output feedback tracking SMC scheme for uncertain linear plants with relative degree one was introduced in [1] where, in lieu of the Nussbaum gain, the controller was based on a switching algorithm driven by an appropriate monitoring function of the output error.

In this paper, we extend the controller of [1] to the case of linear plants with arbitrary relative degree. The proposed solution is based on the compensation of the excess of relative degree by means of a hybrid lead filter [10] which combines a conventional linear lead filter and robust exact differentiators (RED) [11].

The application of the controller to a real DC motor control is included to show the viability of its practical implementation.

Preliminaries: The 2-norm (Euclidean norm) of a vector $x$ and the corresponding induced of a matrix $A$ are denoted by $|x|$, or $|A|$, respectively. The $\mathcal{L}_{\infty e}$ norm of the signal

This work was supported in part by FAPERJ, CNPq and CAPES.

A. J. Peixoto, T. R. Oliveira and L.Hsu are with the Dept. of Electrical Eng./COPPE, Federal University of Rio de Janeiro, Rio de Janeiro, Brazil. [jacoud, tiagoroux, liu] acoep.ufrj.br $x(t) \in \mathbb{R}^{n}$ is defined as $\left\|x_{t, \bar{t}_{0}}\right\|:=\sup _{\bar{t}_{0} \leq \tau \leq t}|x(\tau)|$. For $\bar{t}_{0}=0$, the notation $\left\|x_{t}\right\|:=\sup _{0 \leq \tau \leq t}|x(\tau)|$ is adopted. The symbol " $s$ " represents either the Laplace variable or the differential operator " $d / d t$ ", according to the context.

As in [8], [9], the output of a linear time invariant (LTI) system with transfer function $H(s)$ and input $u$ is given by $H(s) u$. Pure convolution $h(t) * u(t)$ is denoted by $H(s) * u$, where $h(t)$ is the impulse response of $H(s)$. Function classes $\mathcal{K}, \mathcal{K}_{\infty}$ are defined according to [12, p.144].

\section{PRoblem Formulation}

Consider an uncertain SISO LTI plant

$$
y=G_{p}(s)\left[u+d_{e}(t)\right],
$$

where $u$ is the control input, $y$ is the output, $d_{e}(t)$ is a matched input disturbance and $G_{p}(s)$ is a strictly proper transfer function. In addition to the usual assumptions of MRAC (Model Reference Adaptive Control), i.e., the plant is minimum-phase, has known order $n$ and known relative degree [9], we assume that: (A1) the unknown parameters of $G_{p}(s)$ belong to a known compact set; (A2) the input disturbance $d_{e}$ is piecewise continuous and uniformly bounded with a known bound $\bar{d}_{e}(t)$ satisfying $\left|d_{e}(t)\right| \leq \bar{d}_{e}(t) \leq$ $\bar{d}_{\text {sup }}, \forall t \geq 0$, where $\bar{d}_{\text {sup }}>0$ is a constant; (A3) the sign of the high frequency gain $k_{p}:=\lim _{s \rightarrow \infty} s^{n^{*}} G_{p}(s) \neq 0$ is unknown, with $n^{*}$ being the relative degree of $G_{p}$.

The Reference Model is given by

$$
y_{m}=M(s) r=\left(k_{m} / D_{m}(s)\right) r, k_{m}>0,
$$

where the reference signal $r(t)$ is assumed piecewise continuous and uniformly bounded and $D_{m}$ is a monic polynomial of degree $n^{*}$.

The aim is to achieve global stability properties in the sense of uniform signal boundedness and asymptotic output tracking, i.e.,

$$
e_{0}(t)=y(t)-y_{m}(t)
$$

should asymptotically tend to zero (exact tracking).

\section{OUTPUT ERROR EQUATION}

Considering the usual model reference adaptive control (MRAC) approach [9], the output error $e_{0}$ satisfy

$$
e_{0}=\rho^{*} M(s)\left[u-u^{*}\right],
$$

where $\rho^{*}=k_{p} / k_{m}$ and

$$
u^{*}(t):=\theta^{* T} \omega(t)-d_{e}(t)+W_{d}(s) * d_{e}(t)
$$

is the model matching control in the presence of $d_{e}$. The regressor vector $\omega$ is composed by the states of the input 
filter $W_{d}(s)$ (BIBO stable and strictly proper), by the states of output filter, by the plant output $y$ and by the reference signal $r$ [9]. The ideal parameter vector $\theta^{*}$ is unknown but is elementwise bounded by a known constant vector [7].

Since there exists $u^{*}$, in (5), that gives the perfect model following control law, it is reasonable to restrict the class of admissible control laws by the following assumption: (A4) The control law satisfies the inequality (with $k_{\omega}, k_{\delta}>0$ constants)

$$
\forall t \geq 0: \quad|u| \leq k_{\omega}|\omega(t)|+k_{\delta} .
$$

\section{Output Feedback Sliding Mode Controller}

One simple way to approach plants with $n^{*}>1$ consists in reducing the problem to the $n^{*}=1$ case by using lead filters to compensate the excess of relative degree. In the proposed control strategy (Fig.1), a hybrid lead filter named Global Robust Exact Differentiator (GRED) [10] is used. The GRED combines linear $(L / F)$ and nonlinear $\left(L_{\text {red }}\right.$ lead compensation, the latter being constructed with Levant's robust exact differentiators (RED) [11].

The GRED provides a surrogate for the non-causal operator

$$
L(s)=s^{N}+a_{N-1} s^{N-1}+\ldots+a_{0}, \quad N:=n^{*}-1 .
$$

The linear lead filter alone $(L(s) / F(\tau s), F(\tau s)=(\tau s+$ $1)^{N}, \tau>0$ ), leads to global stability, but cannot provide the exact estimate of the $e_{0}$ derivatives. Such filters alone are also well known to lead to control chattering with residual tracking error. On the other hand, REDs used in the block $L_{\text {red }}$, allow the local exact estimate of the $e_{0}$ derivatives. However, when used in a feedback loop, only local exact differentiation can be guaranteed. A convex combination based on a continuous switching law $\alpha(\cdot)$ [10], results in ultimate exact compensation of the relative degree while assuring global stability properties of the closed loop system.

\section{A. Control Law}

According to Fig. 1, the control signal $u$ is defined as:

$$
u= \begin{cases}u^{+}=-f(t) \operatorname{sgn}\left(\tilde{\varepsilon}_{0}\right) & , \quad t \in T^{+}, \\ u^{-}=f(t) \operatorname{sgn}\left(\tilde{\varepsilon}_{0}\right) & , \quad t \in T^{-},\end{cases}
$$

where $\tilde{\varepsilon}_{0}=(1-\alpha) \bar{\varepsilon}_{0}+\alpha \varepsilon_{0}$. As in [1], a monitoring function $\varphi_{m}$ for the auxiliary error $\tilde{\varepsilon}_{0}$ is used to decide when $u$ should be switched from $u^{+}$to $u^{-}$and vice versa, based on the detection of a wrong estimate of $\operatorname{sgn}\left(k_{p}\right)$. The sets $T^{+}$and $T^{-}$satisfy $T^{+} \cup T^{-}=[0, \infty)$ and $T^{+} \cap T^{-}=0$, and both have the form $\left[t_{k}, t_{k+1}\right) \cup \cdots \cup\left[t_{l}, t_{l+1}\right)$. Here, $t_{k}$ or $t_{l}$ denote switching times for $u$.

\section{B. Modulation function}

A possible choice for the modulation function $f(t)$ to satisfy the inequality $f \geq\left|u^{*}\right|$, modulo exponentially decaying terms with $u^{*}$ defined in (5), is given by

$$
f(t)=\left|\bar{\theta}^{T} \omega(t)\right|+\hat{d}_{e}(t)+\delta,
$$

where $\delta>0$ is an arbitrary constant, $\hat{d}_{e}=\hat{W}_{d}(s) \bar{d}_{e}+\bar{d}_{e}$ and $\hat{W}_{d}(s)$ is a first order approximation filter (FOAF) [13, Lemma 2] for $W_{d}(s)$.

The parameter vector $\bar{\theta}^{T}$ is such that $\bar{\theta}_{i}>\max \left\{\left|\theta_{i}^{*}\right|,\left|\theta_{i}^{\dagger}\right|\right\}$, where $\theta^{\dagger}$ is the ideal parameter vector that matches the closed loop system with an unstable reference model $M^{\dagger}(s)$ such that $M^{\dagger} L(s)=k_{m} /\left(s-a_{m}\right)$, with $k_{m}, a_{m}>0$. This will be useful to guarantee that the control direction will be correctly found.

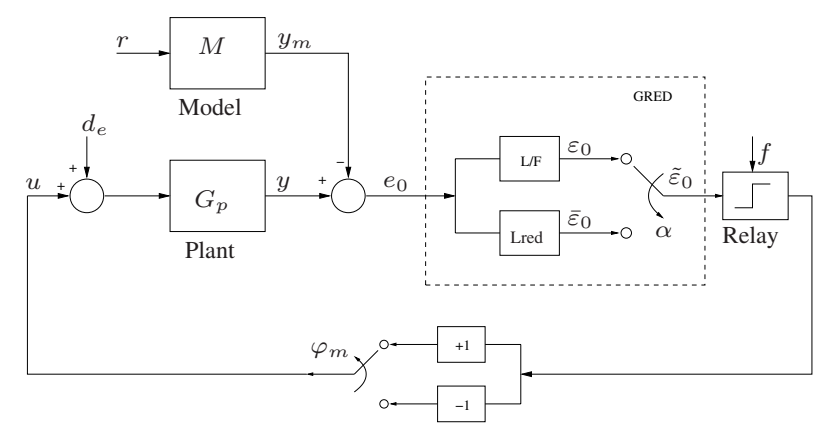

Fig. 1. Sliding mode controller using a hybrid lead filter (GRED) for relative degree compensation and a monitoring function $\varphi_{m}$ to switch the control sign.

\section{Equivalent Structure for the Hybrid Lead Filter}

According to [10, Lemma 2], with an appropriate switching function $\alpha(\cdot)$ the GRED (Fig. 1) is equivalent to a linear lead filter perturbed by a uniformly bounded output measurement disturbance $\beta_{\alpha}$ of order $\tau$ modulo exponentially decaying terms. In order to simplify the analysis, we will postpone its consideration to Subsection VII-A. Thus, in what follows $\tilde{\varepsilon}_{0}=\varepsilon_{0}$.

\section{Lead Filter ERror Equation}

In what follows, we assume that $\tau \in(0, \bar{\tau}]$, where $\bar{\tau}<1$ is some sufficiently small constant. As in high gain observers, lead filters inevitably generate peaking [12] as $\tau \rightarrow 0$. Considering the canonical controllable realization of linear lead filter $L / F$ (Fig. 1), the suitable similar transformation $T:=\operatorname{diag}\left\{\tau^{-N}, \tau^{-(N-1)}, \ldots, \tau^{-1}\right\}$ leads to a peaking free state vector $x_{f}$. Then, peaking appears only at the output $\tau^{N} \varepsilon_{0}=C_{f}(\tau) x_{f}+e_{0}$ where matrix $C_{f}(\tau)$ is finite for $\tau=0$. Let $\left(A_{c}, b_{c}, h_{c}^{T}\right)$ be a stable non-minimal realization of $M(s)$ in (4), with state vector $X_{e}$. From (4), the auxiliary error $\varepsilon_{0}$ (Fig.1) satisfies

$$
\varepsilon_{0}=\rho^{*} M L\left[u-u^{*}\right]+\beta_{\mathcal{U}}+e_{F}^{0},
$$

where

$$
\beta_{\mathcal{U}}:=\rho^{*} M L(s)[1-F(\tau s)] F^{-1}(\tau s) *\left(u-u^{*}\right)
$$

and $e_{F}^{0}$ is an exponentially decreasing term due to the initial conditions $X_{e}(0)$ and $x_{f}(0)$, which can be bounded by

$$
\left|e_{F}^{0}\right| \leq R_{1} e^{-\lambda_{c} t}+\frac{R_{2}}{\tau^{N}} e^{-\frac{t}{\tau}} \leq R_{a} e^{-\lambda_{a}\left(t-t_{e}(\tau)\right)},
$$

where $R_{1}=k_{1}\left|x_{f}(0)\right|, R_{2}=k_{2}\left(\left|X_{e}(0)\right|+\left|x_{f}(0)\right|\right), R_{a}=$ $k_{a}\left(\left|X_{e}(0)\right|+\left|x_{f}(0)\right|\right)$ are positive constants independent of 
$\tau$ and $0<\lambda_{c}<\min _{i}\left\{-R_{e}\left(\lambda_{i}\left[A_{c}\right]\right)\right\}$, where $\left\{\lambda_{i}\left[A_{c}\right]\right\}$ is the spectrum of $A_{c}$ and $0<\lambda_{a}<\min \left(\lambda_{c}, 1 / \bar{\tau}\right)$.

The first inequality in (12) holds $\forall t \geq 0$, while the last one holds only $\forall t \geq t_{e}$ where $t_{e}$ is the peak extinction time, i.e., the smallest time value for which $e^{-\frac{t}{\tau}} / \tau^{N} \leq 1, \forall t \geq t_{e}(\tau)$, is satisfied for a given value of the parameter $\tau \in(0,1]$. It can be concluded that $t_{e}(\tau) \leq \bar{t}_{e}(\tau)$, where $\bar{t}_{e}(\tau) \in \mathcal{K}$ can be obtained from the known upper bounds of the plant parameters.

\section{MOnitoring FunCtion}

The monitoring function is based on the following lemma, which is valid if $\operatorname{sgn}\left(k_{p}\right)$ is correct and if $t \geq \bar{t}_{e}$.

Lemma 1: Consider the I/O relationship

$$
\varepsilon(t)=\bar{M}(s)[u+d(t)]+\pi(t)+\beta(t),
$$

where $\bar{M}(s)=\bar{k} /(s+\bar{\alpha})(\bar{k}, \bar{\alpha}>0), d(t)$ is locally integrable in the sense of Lebesgue (LI) and $\beta(t)$ and $\pi(t)$ are absolutely continuous, $\forall t \geq \bar{t}_{0}$, with $\bar{t}_{0} \geq 0$ being an arbitrary initial time. Assume that $|\pi(t)| \leq R e^{-\lambda\left(t-\bar{t}_{0}\right)}, \forall t \geq$ $\bar{t}_{0}$, where $R, \lambda>0$ are constants. Let $\gamma(t)$ be an absolutely continuous function that satisfies $\left(\forall t \geq \bar{t}_{0}\right)$

$$
\gamma(t) \geq|\beta(t)|+|\pi(t)| \text { and } \quad \frac{d}{d t} \gamma(t) \geq-\bar{\alpha} \gamma(t) .
$$

If $u=-f(t) \operatorname{sgn}(\varepsilon)$, where the modulation function $f(t)$ is LI and satisfies $f(t) \geq|d(t)|, \forall t \geq \bar{t}_{0}$, then the signal $\bar{e}(t):=\varepsilon(t)-\beta(t)-\pi(t)$ is bounded by

$$
|\bar{e}(t)| \leq\left|\varepsilon\left(t_{i}\right)-\beta\left(t_{i}\right)\right| e^{-\bar{\alpha}\left(t-t_{i}\right)}+\gamma(t),
$$

where $t_{i}$ is an arbitrary instant such that $t_{i} \geq \bar{t}_{0}$.

Proof: See the proof of [8, Lemma 2].

Reminding that $\varepsilon_{0}=\beta_{\mathcal{U}}+\bar{e}_{0}+e_{F}^{0}$ then $\left|\varepsilon_{0}\right| \leq\left|\beta_{\mathcal{U}}\right|+\left|\bar{e}_{0}\right|+$ $\left|e_{F}^{0}\right|$. Let $\gamma(t):=\left(R_{a} e^{\bar{\lambda}_{a} \bar{t}_{e}}\right) e^{-\bar{\lambda}_{a} t}+\left\|\left(\beta_{\mathcal{U}}\right)_{t, \bar{t}_{e}}\right\|$, with $\bar{\lambda}_{a}=$ $\min \left\{a_{m}, \lambda_{a}\right\}$ and $M(s)=k_{m} /\left(s+a_{m}\right)\left(a_{m}, k_{m}>0\right)$ for simplicity. Applying Lemma 1 to (10), with $\bar{t}_{0}:=\bar{t}_{e}$ and considering (12), then the following inequality holds

$$
\left|\varepsilon_{0}(t)\right| \leq\left(\left|\varepsilon_{0}\left(t_{k}\right)\right|+\left|\beta_{\mathcal{U}}\left(t_{k}\right)\right|\right) e^{-a_{m}\left(t-t_{k}\right)}+2 \gamma(t),
$$

$\forall t, t_{k}\left(t \geq t_{k} \geq \bar{t}_{e}\right)$. Consider the available signal

$$
\bar{\beta}_{\mathcal{U}}=2 \bar{\rho}^{*} W_{\beta}(s, \tau) * f(t),
$$

where $\bar{\rho}^{*} \geq \rho^{*}$ and $W_{\beta}(s, \tau)$ can be chosen, through partial fraction expansion of $M L(s)[1-F(\tau s)] F^{-1}(\tau s)$, as a sum of two FOAFs, one of them with a fast pole $-1 / \tau$ and such that the induced $\mathcal{L}_{\infty}$ norm of the operator $W_{\beta}(s, \tau)$ is of order $\mathcal{O}(\tau)$. Let

$$
\begin{aligned}
\varphi_{k}(t) & :=\left(\left|\varepsilon_{0}\left(t_{k}\right)\right|+\bar{\beta}_{\mathcal{U}}\left(t_{k}\right)\right) e^{-a_{m}\left(t-t_{k}\right)}+ \\
& +a(k) e^{-\lambda_{c} t}+2\left\|\left(\bar{\beta}_{\mathcal{U}}\right)_{t}\right\|,
\end{aligned}
$$

$\forall t \in\left[t_{k}, t_{k+1}\right)$, where $\lambda_{c}$ is given in (12) and $a(k)$ is any positive monotonically increasing sequence satisfying $a(k) \rightarrow+\infty$ as $k \rightarrow+\infty$. The monitoring function for the arbitrary relative degree case $\varphi_{m}$ is defined by

$$
\varphi_{m}(t):=\varphi_{k}(t), \forall t \in\left[t_{k}, t_{k+1}\right)(\subset[0, \infty)) .
$$

The switching time $t_{k}$ from $u^{-}$to $u^{+}$(or $u^{+}$to $u^{-}$) is defined by

$$
t_{k+1}:= \begin{cases}\min \left\{t>t_{k}:\left|\varepsilon_{0}(t)\right|=\varphi_{k}(t)\right\}, & \text { if it exists } \\ \infty, & \text { otherwise }\end{cases}
$$

where $k \geq 1, t_{0}:=0$ and $t_{1}:=\bar{t}_{e}$. For convenience, $\varphi_{0}=0$, $\forall t \in\left[t_{0}, t_{1}\right)$, see Fig. 2. The following inequality is directly obtained from (19)

$$
\left|\varepsilon_{0}(t)\right| \leq \varphi_{m}(t), \quad \forall t \geq t_{1} .
$$

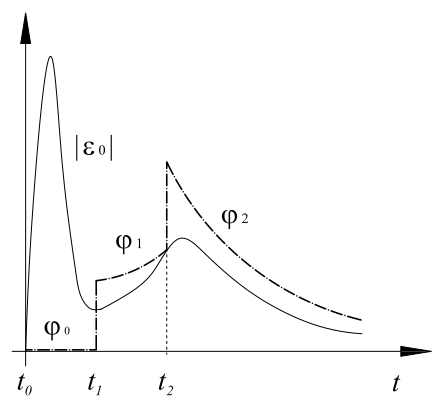

Fig. 2. The trajectories of $\varphi_{m}(t)$ and $\varepsilon_{0}(t)$.

\section{StABILITY ANALYSIS}

In order to fully account for the initial conditions, considering the error system (4), (10), the following state vector $z$ is used

$$
z^{T}:=\left[X_{e}^{T}, x_{f},\left(z^{0}\right)^{T}\right]
$$

where $z^{0}$ denotes the transient state [8] corresponding to $\hat{W}_{d}$ in (9). The following inequality is a consequence of the continuity of the Filippov solutions and the particular state realization associated with $x_{f}$

$$
|z(t)| \leq k_{z 0}|z(0)|+\mathcal{V}(\tau)
$$

$\forall t \in\left[0, t_{e}(\tau)\right], \forall \tau \in\left(0, \tau_{1}\right], 0<\tau_{1} \leq 1$, where $\mathcal{V} \in \mathcal{K}$ and $k_{z 0}>0$ is a constant. It assures that $z$ is peaking free.

The following proposition guarantees boundness of the full state $z$ during the switching of the control direction.

Proposition 1: Assume that (A1)-(A4) hold. Consider the complete error system (4), (8) and (10), with state $z$ defined in (22). Let $t_{k}$ be the last switching time of the monitoring function (19), before the current time $t$. Then, there exists a sufficiently small $\tau_{2}>0$ such that $\forall \tau \in\left(0, \tau_{2}\right]$, the complete error system is bounded by

$$
|z(t)| \leq s_{1}(k)|z(0)|+s_{2}(k) a(k)+s_{3}(k) \mathcal{O}(\tau),
$$

$\forall t \geq 0$, where $s_{i}(k)$ are positive increasing functions of $k$.

Proof: See Appendix .

The main stability and convergence result can be stated in the following theorem (for the linear lead filter compensation): 
Theorem 1: Assume that (A1)-(A4) hold, the modulation function satisfies (9) and $M L(s)=k_{m} /\left(s+a_{m}\right)$ with $\left(k_{m}, a_{m}>0\right)$. Then, for sufficiently small $\tau>0$, the switchings of the control sign, driven by the monitoring function (19), stop after a finite number of switchings and the complete error system (4), (8) and (10), with state $z(22)$, is globally asymptotically stable with respect to a compact set and ultimately exponentially convergent to a residual set, both sets being independent of the initial conditions. The residual set is contained in a $\mathcal{V}(\tau)$-ball, with $\mathcal{V}(\tau) \in \mathcal{K}$.

\section{Proof: See Appendix.}

Note that the results of stability with respect to a compact set, not necessarily small, accounts for the initial transient while the monitoring function has not yet stopped. This means that, even if the initial errors are very small, the initial transient may not be correspondingly small.

\section{A. Chattering Avoidance and Exact Tracking}

In Fig. 1, the block $L_{\text {red }}$ represents the "exact lead filter" which implements the operator $L(s)$ by using RED. This allows the realization of ideal sliding mode, thus eliminating the chattering caused by the lag present in the lead filter. The linear filter allows global and asymptotic convergence to some neighbourhood of the error space origin (Theorem 1) while the nonlinear filter allows ideal sliding mode which is reached asymptotically or in finite time. For illustration, we give the RED algorithm to compute the first derivative of a given signal $e_{0}$ :

$$
\begin{aligned}
\dot{\eta}_{0} & =\eta_{1} \\
\dot{v} & =-\mu_{1} \operatorname{sgn}\left(\eta_{0}-e_{0}\right), \\
\eta_{1} & =v-\mu_{0}\left|\eta_{0}-e_{0}\right|^{\frac{1}{2}} \operatorname{sgn}\left(\eta_{0}-e_{0}\right),
\end{aligned}
$$

which provides $\eta_{0}(t) \rightarrow e_{0}(t), \eta_{1}(t) \rightarrow \dot{e}_{0}(t)$. Higher order differentiators can be found in [11]. Let $\tilde{\beta}_{\mathcal{U}}(t)=\beta_{\alpha}+\bar{\beta}_{\mathcal{U}}(t)$. We can now state the stability and exact tracking result.

Corollary 1: With the hybrid lead filter and the monitoring function defined with respect to the auxiliary error $\tilde{\varepsilon}_{0}$ (see Fig. 1), according to

$$
\begin{aligned}
\varphi_{k}(t) & :=\left(\left|\tilde{\varepsilon}_{0}\left(t_{k}\right)\right|+\tilde{\beta}_{\mathcal{U}}\left(t_{k}\right)\right) e^{-a_{m}\left(t-t_{k}\right)}+ \\
& +a(k) e^{-\lambda_{c} t}+2\left\|\left(\tilde{\beta}_{\mathcal{U}}\right)_{t}\right\|,
\end{aligned}
$$

all results of the Theorem 1 hold. Moreover, for an appropriate modulation function, exact tracking is achieved in finite time or at least exponentially and the control sign switching stops at the correct sign.

\section{Proof: See Appendix.}

\section{EXPERIMENTAL RESULTS}

In this section, experiments with a DC motor are presented in order to verify the applicability of the proposed strategy to real systems. The experiments were performed using a laboratory prototype based on a DC motor 2342024CR from MicroMo Electronics, Inc., with built in gear box (1:43), armature voltage controlled. The control algorithm was implemented on a DSP motion control board (Arcs Inc.) hosted in a PC which contains an integrated encoder input as well as a 12-bits digital-to-analog converter to drive a linear power driver. The sampling frequency was $2.5 \mathrm{kHz}$. The motor angular position was measured using an incremental optical encoder having resolution 1000 pulses per turn (ppt). The resolution of the load angular position is $172000 \mathrm{ppt}$ due to the gear box and the electronics on the card.

The following nominal relative degree two model of the DC motor is used, neglecting the small electric time constant

$$
G_{p}(s)=\frac{y}{u}=\frac{20 k_{p}}{s(s+10)},
$$

where $y$ is the angular position in degrees, $k_{p} \in[30,50]$ but can have reversed sign and the armature voltage is given by $u$.

Two experiments are discussed here. In the first one, the objective is to validate the performance of the proposed controller for large initial tracking error as well as with initially wrong control direction. The control direction is changed in order to check the recovery capability of the controller under a change of the control direction "on-thefly". Several such changes were made during this experiment. The aim of the second experiment is to evaluate the practical advantage of RED $(\alpha=0)$, compared to a linear lead filter, in a real application.

In both experiments the reference model is $M(s)=$ $\frac{20}{(s+5)(s+20)}$ and the linear lead filter is given by $\frac{L}{F}=\frac{(s+5)}{(\tau s+1)}$, with $\tau=0.002$. The nonlinear lead filter is implemented as in (25) with $\mu_{0}=100$ and $\mu_{1}=2500$. The switching function $\alpha$ of the hybrid lead filter was chosen such that $\epsilon_{M}=80$ and $c=0$. The monitoring function is obtained from (19) with $a(k)=k+1, a_{m}=20, \bar{\lambda}_{c}=1$. In order to counteract the disturbance $\beta_{\alpha}$ introduced by the nonlinear lead filter (see [10]), a constant of 80 was added to the term $\bar{\beta}_{\mathcal{U}}$. In addition, a constant of 10 was also added to the monitoring function to reduce spurious modifications in the control direction estimate due to measurement noise. In order to simplify the control implementation, a constant modulation function was chosen to be $f(t)=5$ which is sufficient enough to guarantee the model following.

In what follows, we discuss in detail the results of each experiment.

\section{A. Experiment I}

In this first experiment the reference signal is a sinusoid such that $y_{m}$ has amplitude $50^{\circ}$ and frequency $0.5 \mathrm{~Hz}$. The plant initial condition are $y(0)=100^{\circ}, \dot{y}(0)=0$ and the feedback is initially positive at $t=0$ (wrong control direction). Several changes in the control direction were artificially introduced during the experiment as explained above. The initial response, up to $t=7 \mathrm{~s}$, is shown in Fig. 3(a). The transient responses to changes in the control direction were quite satisfactory (a transient error of less than $21^{\circ}$ ), see Fig. 3(b). The changes of control directions can be noted from the spikes in the tracking error. Fig. 4 is also plotted the monitoring function $\varphi_{m}$ and the monitoring error $\tilde{\varepsilon}_{0}$ (see Fig. 1). Note that the time range in Fig. 4 (b) is larger, to stress that the nonlinear lead filter is ultimately chosen $(\alpha=0)$. 

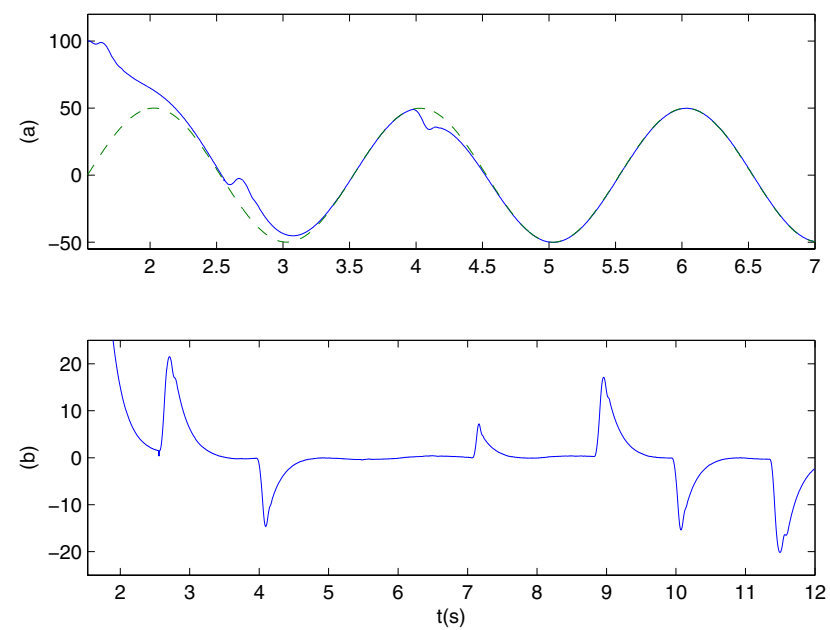

Fig. 3. Experiment I: (a) initial plant output $y$ (solid) (degrees) and reference model output $y_{m}$ (dashed) and (b) tracking error $e_{0}$ (degrees) with several changes in control direction (at the spikes).
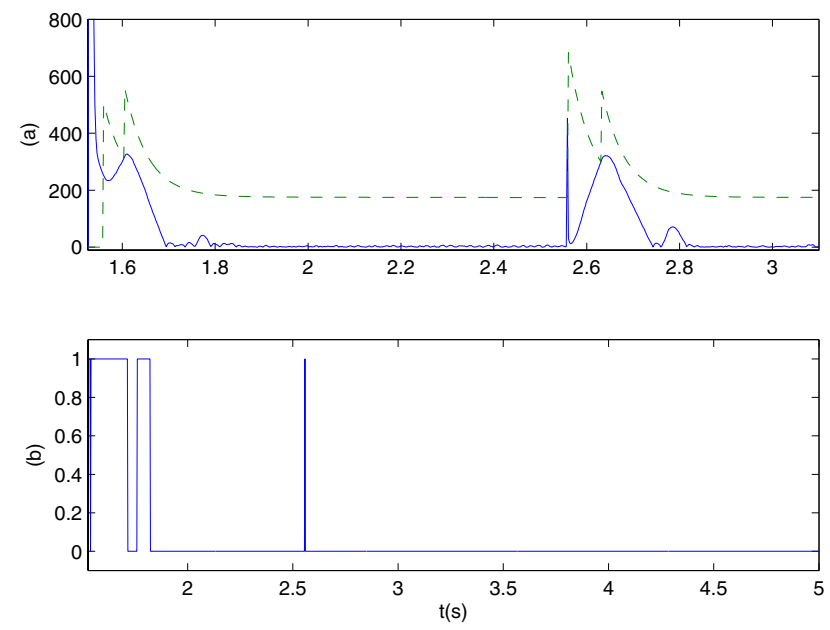

Fig. 4. Experiment I: (a) monitoring function $\varphi_{m}$ (dash) and the auxiliary error $\tilde{\varepsilon}_{0}$ (solid) and (b) switching function $\alpha: \alpha=1$ (linear lead filter) and $\alpha=0$ (nonlinear lead filter).

\section{B. Experiment II}

In this experiment no changes are made in the control direction. In Fig. 5(a), we start with the linear lead filter. Then for $t \in[16,26]$, we manually switch to the nonlinear lead filter $(\alpha=0)$ and then finally we switch again to the linear lead filter. One can clearly note the better performance of the RED based (nonlinear) filter and the performance degradation that results due to the phase lag introduced by the linear lead filter, with $\tau=0.002$ (this parameter was experimentally tuned as small as possible so that the control chattering was acceptable).

Fig. 5(b) and (c) correspond to the response of the system to step changes in the reference input. For $t \in[14,28]$ and $t \in[37,45]$, only the nonlinear lead filter is used, while in the remaining intervals of time, the linear lead filter is used. A noticeable control chattering results in the latter case, in steady state following a step input. In contrast, the chattering is practically eliminated in the case of the nonlinear lead filter. Thus, a remarkably superior regulation performance is observed when using the RED based nonlinear lead filter.
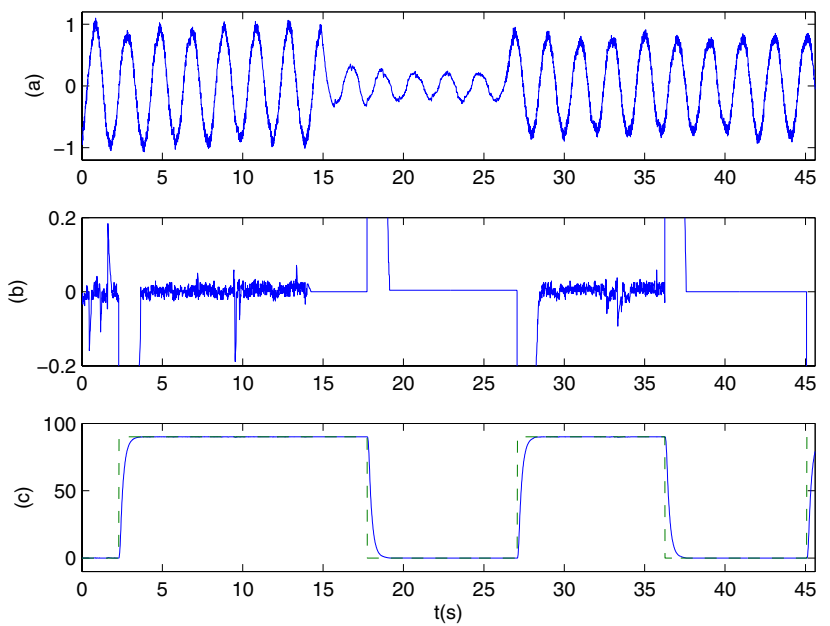

Fig. 5. Experiment II: using linear lead filter or nonlinear RED based nonlinear lead filter; (a) output error $e_{0}$ (tracking a sinusoid)(degrees) ; (b) output error $e_{0}$ in response to step inputs; (c) $y$ (solid)(degrees) and reference step inputs (dashed).

\section{CONCLUSIONS}

An output-feedback model-reference sliding mode controller was developed for linear uncertain systems with unknown high frequency gain sign and arbitrary relative degree generalizing the VS-MRAC controller introduced in [1]. Experiments on a DC motor position control were presented to show the practical applicability of the proposed controller in real world conditions.

The resulting controller leads to global asymptotic stability with respect to some compact set and ultimate exponential or finite time convergence of the tracking error to zero. The controller has led to quite reasonable transient behavior and the nonlinear exact differentiator has led to better performance in comparison with conventional linear differentiators.

\section{ACKNOWLEDGMENTS}

The authors acknowledge the partial support of CNPq, FAPERJ and CAPES (Brazil).

\section{APPENDIX}

\section{A. Proof of Proposition 1}

In what follows, $k_{i}$ denotes positive constants that depend only on the plant-controller parameters and $\Psi_{i}(\cdot)$ denotes functions of class $\mathcal{K}_{\infty}$. From (18), (19) and (21), one has

$$
\left\|\left(\varepsilon_{0}\right)_{t, t_{1}}\right\| \leq\left|\varepsilon_{0}\left(t_{p}\right)\right|+a(k)+3\left\|\left(\bar{\beta}_{\mathcal{U}}\right)_{t}\right\|, \quad\left(\forall t \geq t_{1}\right),
$$

where $k \geq 1, p=\operatorname{argmax}_{i \in\{1,2, \ldots, k\}}\left|\varepsilon_{0}\left(t_{i}\right)\right|$ and $t \in$ $\left[t_{k}, t_{k+1}\right]$.

From (12), one can conclude that $\left\|\left(e_{F}^{0}\right)_{t, t_{1}}\right\| \leq k_{1}|z(0)|$, with $z$ defined in (22). Thus, reminding that $\bar{e}_{0}=\varepsilon_{0}-\beta_{\mathcal{U}}-$ $e_{F}^{0}$ the following inequality holds $\left(\forall t \geq t_{1}\right)$

$$
\left\|\left(\bar{e}_{0}\right)_{t, t_{1}}\right\| \leq\left|\bar{e}_{0}\left(t_{p}\right)\right|+a(k)+2 k_{1}|z(0)|+5\left\|\left(\bar{\beta}_{\mathcal{U}}\right)_{t}\right\| .
$$


Now, since $X_{e}$ is the state of a stable non-minimal realization of the transfer function $M L(s)=k_{m} /\left(s+a_{m}\right) ; k_{m}, a_{m}>$ $0)$, it is possible to linearly transform $X_{e}$ to a new state $\bar{X}_{e}=P X_{e}=\left[\begin{array}{ll}\bar{e}_{0} & \bar{X}_{e 2}^{T}\end{array}\right]^{T}$, where $\bar{X}_{e 2}$ is exponentially decaying. Moreover, since $x_{f}$ is driven by the tracking error $e_{0}=h_{c}^{T} X_{e}$, and taking into account (23), an upper bound similar to (29) is also valid for $z$ (22), i.e., the state $z$ satisfies $(\forall t \geq 0)$

$$
\left\|(z)_{t}\right\| \leq k_{2}|z(0)|+k_{3}\left|z\left(t_{p}\right)\right|+k_{4} a(k)+k_{5}\left\|\left(\bar{\beta}_{\mathcal{U}}\right)_{t}\right\|+\mathcal{O}(\tau) .
$$

Now, from (17), the small norm property of $W_{\beta}(s, \tau)$ and since $f(t)$ is given by (9) with $\omega$ affinely bounded by $\left\|X_{e}\right\|$ [9], one has

$$
\left\|\left(\bar{\beta}_{\mathcal{U}}\right)_{t}\right\| \leq \tau k_{6}\left(\left\|(z)_{t}\right\|\right)+\tau k_{7}
$$

Moreover, from (30) and (31), for $\tau<1 /\left(k_{2} k_{5}\right)$, we get $(\forall t \geq 0)$

$$
\left\|(z)_{t}\right\| \leq k_{8}\left|z\left(t_{p}\right)\right|+k_{9} a(k)+k_{10}|z(0)|+\mathcal{O}(\tau) .
$$

Then, noting that $\left|z\left(t_{p}\right)\right| \leq \sum_{i=1}^{k}\left|z\left(t_{i}\right)\right|$, the following recursive inequality follows

$$
\left|z\left(t_{k+1}\right)\right| \leq k_{8} \sum_{i=1}^{k}\left|z\left(t_{i}\right)\right|+k_{9} a(k)+k_{10}|z(0)|+\mathcal{O}(\tau)
$$

whereby (24) results.

\section{B. Proof of Theorem 1}

The monitoring function has to stop switching after some finite $k=k^{*}$. Indeed, since $a(k)$ increases unboundedly as $k \rightarrow \infty$, there is a finite value $k_{1}$ such that for $k \geq k_{1}$ one has $a(k) \geq\left(2 R_{a} e^{\bar{\lambda}_{a} \bar{t}_{e}}\right)$ (see (16)). It is not difficult to conclude that $k^{*}$ can be related to $R_{0}:=|z(0)|$ reminding that $R_{a}=k_{a}|z(0)|$ by definition. In fact, one can write

$$
k^{*} \leq \mathcal{V}_{k}\left(R_{0}\right)+k_{0},
$$

where $k_{0}>0$ is a constant and $\mathcal{V}_{k} \in \mathcal{K}_{\infty}$.

Now, from Proposition 1, it follows that, for $\tau$ sufficiently small

$$
|z(t)| \leq \mathcal{V}_{z}\left(R_{0}\right)+c_{z}
$$

where, $c_{z}>0$ is a constant and $\mathcal{V}_{z} \in \mathcal{K}_{\infty}$. Thus, if $R>\mathcal{V}_{z}\left(R_{0}\right)+c_{z}$, then $|z(t)|<R, \forall t \geq 0$. Thus, stability with respect to the ball of radius $c_{z}$ is guaranteed for initial conditions in the $R_{0}$-ball. Since $R_{0}$ can be chosen arbitrarily large, global stability is concluded.

If the control direction is correctly found at $k=k^{*}$, then $|z(t)| \rightarrow \mathcal{V}(\tau)$ exponentially $(\mathcal{V} \in \mathcal{K})$, as $t \rightarrow+\infty$, according to [8, Theorem 2]. Otherwise, the control pursues with wrong direction while all signals remain uniformly bounded $\forall t>t_{k^{*}}$. Indeed, by Proposition 1, for sufficiently small $\tau$, all signals remain uniformly bounded after the last switching $k^{*}$. Since we have chosen a modulation function capable of making the closed loop unstable if wrong control direction is applied, one can show that there exists a sign indefinite quadratic function $V(z)$ which has positive time derivative outside a compact set around the error space origin. According to a stability analysis similar to that of Cetaev's Instability Theorem [12], this implies that the system must enter a residual set where $\left|X_{e}(t)\right|<\mathcal{V}(\tau)$ after some finite time. A rigorous proof follows closely the method of [14]. In addition, reminding that the state $x_{f}$ is driven by the signal $\bar{e}_{0}=h_{L}^{T} X_{e}$, then the convergence of $X_{e}$ implies $\left|x_{f}(t)\right|,|z(t)|<\mathcal{V}(\tau), \forall t$ after some finite time.

\section{Proof of Corollary 1}

The hybrid lead filter only introduces a disturbance $\beta_{\alpha}$ [10] which is norm-bounded by a design constant of order $\mathcal{O}(\tau)$, modulo decaying exponential terms which can be embedded in $e_{F}^{0}$ (12). This constant bound can be simply added to the bound of $\bar{\beta}_{\mathcal{U}}$ given in (17). The monitoring function is redefined in an appropriate way in order to monitor the perturbed auxiliary signal $\tilde{\varepsilon}_{0}$. The exact differentiator takes over since the error state enters the residual set (Theorem 1). Then, the system becomes exactly a relative degree one case. The control sign must be correct after the last switching at $k=k^{*}$. Indeed, we had the incorrect control sign, then by using a Cetaev's Theorem argument, we could show that $e_{0}$ would not remain in the residual set of Theorem 1 , which is a contradiction.

\section{REFERENCES}

[1] L. Yan, L. Hsu, R. R. Costa and F. Lizarralde, "Variable Structure Model Reference Adaptive Control for Systems with Unknown High Frequency Gain", 2003 Conference on Decision and Control, Hawaii, pp. 3525-3530, 2003.

[2] R. D. Nussbaum, "Some results on a conjecture in parameter adaptive control", Syst. Contr. Lett., vol. 3, pp. 243-246, 1983.

[3] D. R. Mudgett and A.S. Morse, "Adaptive stabilization of linear systems with unknown high frequency gains", IEEE Trans. Automat. Contr., vol. 30, pp. 549-554, 1985.

[4] M. Fu and B. R. Barmish, "Adaptive stabilization of linear systems via switching control", IEEE Trans. Aut. Contr., vol. 31, no. 12, pp. 1097$1103,1986$.

[5] S. Drakunov, "Sliding Mode Control of the Systems with Uncertain Direction of Control Vector", Conference on Decision and Control, San Antonio, pp. 2477-2478, 1993.

[6] G. Bartolini, A. Ferrara and L. Giacomini, "A Switching Controller for Systems with Hard Uncertainties", IEEE Trans. on Circuits and Sytems, vol. 50, no. 8, pp. 984-990, 2003.

[7] L. Hsu, A. D. Araújo, and R. R. Costa, "Analysis and design of I/O based variable structure adaptive control," IEEE Trans. Aut. Contr., vol. 39, no. 1, pp. 4-21, 1994.

[8] L. Hsu, F. Lizarralde, and A. D. Araújo, "New results on outputfeedback variable structure adaptive control: design and stability analysis," IEEE Trans. Aut. Contr., vol. 42, no. 3, pp. 386-393, 1997.

[9] P. A. Ioannou and J. Sun, Robust Adaptive Control. Prentice-Hall, 1996.

[10] E. V. L. Nunes, L. Hsu and F. Lizarralde, "Globally Stable OutputFeedback Sliding Mode Control with Asymptotic Exact Tracking”, in American Control Conference, Boston, Ma, 2004, pp. 638-643.

[11] A. Levant, "Higher-order sliding modes, differentiation and outputfeedback control," Int. J. Contr., vol. 76, no. 9, pp. 924-941, 2003.

[12] H. K. Khalil. Nonlinear Systems. Prentice Hall, $3^{\text {nd }}$ edition, 2002.

[13] L. Hsu, R. R. Costa, and J. P. V. S. Cunha. Model-reference outputfeedback sliding mode controller for a class of multivariable nonlinear systems. Asian Journal of Control, 5(4):543-556, 2003.

[14] R. R. Costa and L. Hsu. Robustness of VS-MRAC with respect to unmodelled dynamics and external disturbances. International Journal of Adaptive Control and Signal Processing, 6:19-33, 1992. 